Research Paper

\title{
THRB Genetic Polymorphisms Can Predict Severe Myelotoxicity aiter De- finitive Chemoradiotherapy in Patients with Esophageal Squamous Cell Carcinoma
}

\author{
Ikuya Miki1,2, Tsutomu Nakamura3,4, Akiko Kuwahara3,5, Motohiro Yamamori3,5, Kohshi Nishiguchi,6, \\ Takao Tamura1, Tatsuya Okuno', Hideaki Omatsu³ ${ }^{3}$, Shigeto Mizuno ${ }^{2}$, Midori Hirai ${ }^{3}$, Takeshi Azuma ${ }^{1}$, and \\ Toshiyuki Sakaeda3,7凶
}

1. Department of Gastroenterology, Kobe University Graduate School of Medicine, Kobe 650-0017, Japan;

2. Department of Medical Pharmaceutics, Kobe Pharmaceutical University, Kobe 658-8558, Japan;

3. Department of Hospital Pharmacy, School of Medicine, Kobe University, Kobe 650-0017, Japan;

4. Department of Pharmaceutical Health Care, Faculty of Pharmaceutical Sciences, Himeji Dokkyo University, Himeji 670-8524, Japan;

5. School of Pharmacy and Pharmaceutical Sciences, Mukogawa Women's University, Nishinomiya 663-8179, Japan;

6. Department of Clinical Pharmacy, Kyoto Pharmaceutical University, Kyoto 607-8414, Japan;

7. Graduate School of Pharmaceutical Sciences, Kyoto University, Kyoto 606-8501, Japan.

Corresponding author: Toshiyuki Sakaeda, Ph.D., Center for Integrative Education in Pharmacy and Pharmaceutical Sciences, Graduate School of Pharmaceutical Sciences, Kyoto University, Kyoto 606-8501, Japan, Tel: +81-75-753-9560, Fax: +81-75-753-9253, e-mail: sakaedat@pharm.kyoto-u.ac.jp.

( ) Ivyspring International Publisher. This is an open-access article distributed under the terms of the Creative Commons License (http://creativecommons.org/ licenses/by-nc-nd/3.0/). Reproduction is permitted for personal, noncommercial use, provided that the article is in whole, unmodified, and properly cited.

Received: 2012.08.21; Accepted: 2012.10.15; Published: 2012.10.23

\begin{abstract}
Objective: Chemotherapy-related toxicities are difficult to predict before treatment. In this study, we investigated whether thyroid hormone receptor beta (THRB) genetic polymorphisms can serve as a potential biomarker in patients with esophageal squamous cell carcinoma (ESCC).

Methods: Forty-nine Japanese patients with ESCC who received a definitive chemoradiotherapy (CRT) with 5-fluorouracil and cisplatin in conjunction with concurrent irradiation were retrospectively analyzed. Severe acute toxicities, including leukopenia, stomatitis, and cheilitis, were evaluated according to 6 single nucleotide polymorphisms (SNPs) in the gene; the intronic SNPs of rs7635707 G/T, rs6787255 A/C, rs $9812034 \mathrm{G} / \mathrm{T}$, and rs $9310738 \mathrm{C} / \mathrm{T}$ and the SNPs in the 3'-untranslated region (3'-UTR) of rs844107 C/T and rs/349265 G/A.

Results: Distribution of the 4 intronic SNPs, but not the 2 SNPs in the 3'-UTR, showed a significant difference between patients with and without severe acute leukopenia. Stomatitis and cheilitis were not associated with any of the 6 analyzed SNPs. Frequency of haplotype of the 4 intronic SNPs reached approximately $97 \%$ with the 2 major haplotypes G-A-G-C (73.4\%) and T-C-T-T (23.5\%).

Conclusions: THRB intronic SNPs can provide useful information on CRT-related severe myelotoxicity in patients with ESCC. Future studies will be needed to confirm these findings.
\end{abstract}

Key words: esophageal squamous cell carcinoma, thyroid hormone receptor beta, chemoradiotherapy, severe acute toxicity, prognosis. 


\section{Introduction}

Esophageal cancer is a common gastrointestinal malignancy that continues to remain virulent. In Japan, 17,000 new cases of esophageal cancer are diagnosed each year, with poor prognosis [1]. The incidence of esophageal squamous cell carcinoma (ESCC) is higher than that of esophageal adenocarcinoma in east Asian countries, including Japan, whereas the incidence of esophageal adenocarcinoma is high in north America, the United kingdom/Ireland, and Australia [2]. The standard therapeutic approach for ESCC has traditionally been radical surgery with node dissection; however, recent advances in chemoradiotherapy (CRT) have been widely accepted as a promising modality in both neoadjuvant and definitive settings for patients with ESCC [3, 4]. A definitive CRT with 5-fluorouracil (5-FU) and cisplatin (CDDP) has been proposed as an organ-preserving treatment $[5,6]$, and clinical evidence supporting its efficacy has been collected in T4 and/or M1 lymph node [5, 6], and later stage II/III [7]. However, the effectiveness of cytotoxic chemotherapy is often limited by serious toxicities. In this definitive 5-FU/CDDP-based CRT, myelosuppression, stomatitis, and cheilitis are some of the most common toxicities [5-7], and severe acute leukopenia constitutes a life-threatening risk in more than a few patients. Identification of patients who can achieve a better treatment course would allow oncologists to maximize therapeutic benefits and minimize toxicities.

Genetic constitution is an important cause of individual variations in the tolerance to chemotherapeutic regimens. Because of the narrow therapeutic index of chemotherapeutic agents, pharmacogenomics has drawn increasing attention in the field of tailored medicine, ranging from gene expression profiles in tumor tissues to genetic signatures, including single nucleotide polymorphisms (SNPs) [3, $8,9]$. The application of SNP genotyping to anticancer therapy is an attractive approach, and serious toxicities have been explained by SNPs in well-known drug-metabolizing enzyme- or drug-transporterrelated genes $[3,8,9]$. However, severe toxicities may have complicated associations with a large variety of genetic mutations; therefore, identification of novel markers using a different concept will be needed.

As a candidate gene approach, we focused on the thyroid hormone receptor beta gene (gene symbol: THRB, gene ID: 7068). Thyroid hormones (THs) play indispensable roles in differentiation, development, and metabolism in various tissues [10], and exert their actions by binding to thyroid hormone receptors (TRs) $[10,11]$. TRs belong to the nuclear hormone re- ceptor superfamily of transcription factors, which generate multiple isoforms by alternative promoter usage and splicing [12]. TRs are widely expressed in different organs and tissues, and TR expression is tissue-dependent and developmentally regulated [13, 14]. THs are thought to be associated with myeloid cell proliferation, given their influence on bone marrow function [15]. Extensive investigations have provided new insights into hematopoietic regulation by THs and TRs [16-18]. TRs are encoded by 2 genes, THRA and THRB, which are located on chromosomes 17 and 3, respectively [19]. Recent studies have revealed their unique functions in radiosensitivity, hepatic proliferation, and renal function [20-24]. In cancer patients receiving radiation therapy with or without chemotherapy, leukopenia and oral mucositis are the commonly detected severe toxicities $[25,26]$. Collectively, the information led us to postulate that $T H R B$ polymorphisms could have predictive value for CRT-related toxicities, and in this study, we retrospectively investigated whether THRB SNPs can predict severe acute toxicities after a definitive 5-FU/CDDP-based CRT in Japanese patients with ESCC.

\section{Materials and Methods}

\section{Ethics statements}

A series of studies has been performed to evaluate the effects of genetic polymorphisms on clinical response 1 month after or severe acute toxicities during treatment with a definitive 5-FU/CDDP-based CRT in Japanese patients with ESCC [27-30]. These studies were conducted with the authorization of the institutional review board (IRB) and followed the medical research council guidelines of Kobe University. All the patients analyzed agreed to the studies and preservation of genomic DNA for future investigations, and additional studies were again authorized by the IRB and followed the guidelines of Kobe University. Written informed consent was obtained from all participants prior to genotyping.

\section{Patients}

The subjects were recruited from the patients diagnosed with ESCC and treated with a definitive CRT at Kobe University Hospital during 2003-2006. Throughout this period, the treatment regimen was standardized at our institution as described below, and was employed for those who were medically unfit for an operation or refused an operation.

In the present study, 49 patients analyzed were on the basis of the following criteria: 1) clinical stage $\mathrm{T} 1$ to $\mathrm{T} 4, \mathrm{~N} 0$ or $\mathrm{N} 1$, and M0 or M1 according to the 
International Union Against Cancer tumor-node-metastasis (TNM) classification; 2) age less than 85 years; 3) an Eastern Cooperative Oncology Group performance status of 0 to $2 ; 4$ ) adequate bone marrow, renal, and hepatic function; 5) no prior chemotherapy; 6) no severe medical complications; 7) no other active malignancies (except early cancer) and 8) availability of written informed consent, as described above. The tumors were histologically confirmed to be primary, and no patients with recurrence were included in this study.

\section{Protocol}

A course consisted of the continuous infusion of $5-\mathrm{FU}$ at $400 \mathrm{mg} / \mathrm{m}^{2} /$ day for days $1-5$ and $8-12$, the infusion of CDDP at $40 \mathrm{mg} / \mathrm{m}^{2} /$ day on days 1 and 8 , and the radiation at $2 \mathrm{~Gy} /$ day on days $1-5,8-12$, and 15-19 (a total dose of $60 \mathrm{~Gy}$ in 30 fractions). This schedule was repeated twice every 5 weeks (Fig. 1). When detecting grade $3 / 4$ hematological toxicities, chemotherapy was withheld until the blood cell counts recovered beyond the critical level, and then a reduced dose was resumed. For fever higher than grade 2, chemotherapy was withheld pending improvement. For renal toxicity, the subsequent dose of CDDP was reduced depending on the degree of toxicity. Radiotherapy was also withheld during the time when the chemotherapy was withheld for severe hematological and/or non-hematological toxicities. In principle, the total radiation dose was not reduced.

\section{Severe acute toxicities}

Leukopenia, stomatitis, and cheilitis, which are the commonly detected severe acute toxicities, were evaluated using criteria defined by the Japan Clinical Oncology Group. These criteria are based on the National Cancer Institute Common Toxicity Criteria. Toxicity was assessed on a 2- to 3-day basis during the CRT and a subsequent hospitalization period and on every visit after the completion of CRT. Episodes of leukopenia, stomatitis, and cheilitis during the first 2 courses and subsequent 2 weeks (until day 70) were recorded as acute toxicities and those of grade 3 or higher as severe acute toxicities.

\section{Genomic DNA extraction and THRB geno- typing}

Genomic DNA was isolated from whole blood using a Taq-Man ${ }^{\circledR}$ Sample-to-SNPTM kit (Applied Biosystems, Foster City, CA, USA), according to the manufacturer's directions, and stored at $-80^{\circ} \mathrm{C}$ until analysis. Genetic polymorphisms of THRB were determined in a TaqMan ${ }^{\circledR}$ MGB probe-based polymerase chain reaction (PCR) using the StepOne ${ }^{\mathrm{TM}}$ real-time PCR system (Applied Biosystems) and TaqMan ${ }^{\circledR}$ SNP genotyping assays (Applied Biosystems). The PCR was carried out according to the manufacturer's protocol. For each set of reactions, the negative controls containing $\mathrm{H}_{2} \mathrm{O}$ instead of DNA were added to detect contamination.

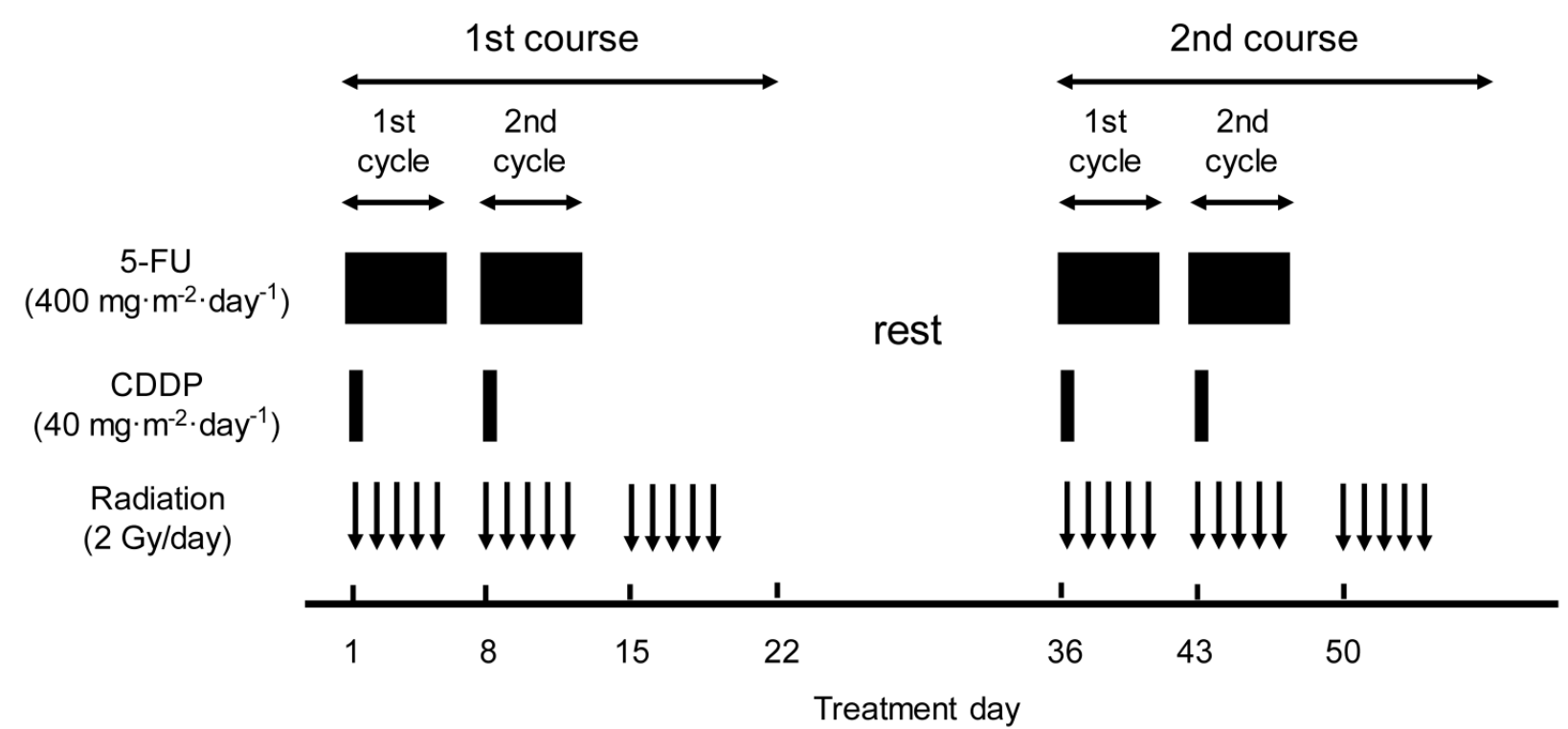

Figure I. Treatment schedule of a definitive 5-FU/CDDP-based chemoradiotherapy. 5-FU, 5-fluorouracil; CDDP, cisplatin. 


\section{SNP selection}

The human THRB gene expresses 2 isoforms, TR $\beta 1$ and TR $\beta 2$, in developmental and tissue-specific patterns by alternative splicing [12]. TR $\beta 1$ and $\beta 2$ differ only in their $\mathrm{N}$ termini. TR $\beta 2$ exon 1 is located in intron 4 of TR $\beta 1$ with the other exons being identical. The 2 isoforms share a common DNA-binding domain and ligand-binding domain. TR $\beta 2$ is restricted to the central nervous system, whereas TR $\beta 1$ is widely expressed. Because only a little is known about the mechanisms underlying their expression, we constructed a genetic concept based on TR $\beta 1$. To date, 4 TR $\beta$ mRNA variants have been identified, and all encode a common protein and have a common non-coding exon 2 (Fig. 2), which led to the hypothesis that SNPs in introns adjacent to exon 2 may influence TR $\beta$ mRNA and protein expression. Therefore, candidate SNPs were selected according to the following criteria: 1) Minor allelic frequencies of the SNP were $>40 \%$ (the frequency of chemotherapy-induced myelotoxicity) in the National Center for Biotechnology Information (NCBI) variation database (dbSNP) [31], and 2) the SNP was located in the adjacent intron, within $10 \mathrm{~kb}$ from the $5^{\prime}$ - or $3^{\prime}$-end of the common exon 2. The allele frequency was related to genetic variations of the SNPs studied and the reference sequences were adopted on the basis of the NCBI dbSNP database. Four SNPs, reference SNP (rs) 7635707, rs6787255, rs9812034, and rs9310738, met the selection criteria described above. The SNPs rs7635707 and rs6787255 are located in intron 1, and rs9812034 and rs9310738 in intron 2. Considering a possible role in TR $\beta$ mRNA stability, additional candidate SNPs in the $3^{\prime}$-untranslated region (3'-UTR), rs844107 and rs1349265, were also selected. The positional relationships of the 6 SNPs analyzed are shown in Figure 2.

\section{Haplotype analysis}

Haplotype frequencies of the SNPs of rs7635707 G/T, rs6787255 A/C, rs9812034 G/T and rs9310738 $\mathrm{C} / \mathrm{T}$ were estimated. The haplotypes were statistically inferred with a fair degree of precision using an algorithm based on Bayesian inference, PHASE version 2.1. Haplotypes were inferred by running PHASE 10 times, and the relative standard deviation (SD) of their frequencies was $0.1 \%$ or less of the mean value $[32,33]$. The estimated haplotype frequencies are expressed as the average population haplotype frequencies for the whole sample, as described previously [34, 35].

\section{Data analysis and statistics}

All values represent mean \pm standard deviation (SD). The associations between clinical characteristics, including each genotype in THRB, and severe acute toxicities were analyzed with Chi-square test or Fisher's exact test. $P$-values $<0.05$ (2-tailed) were considered significant.

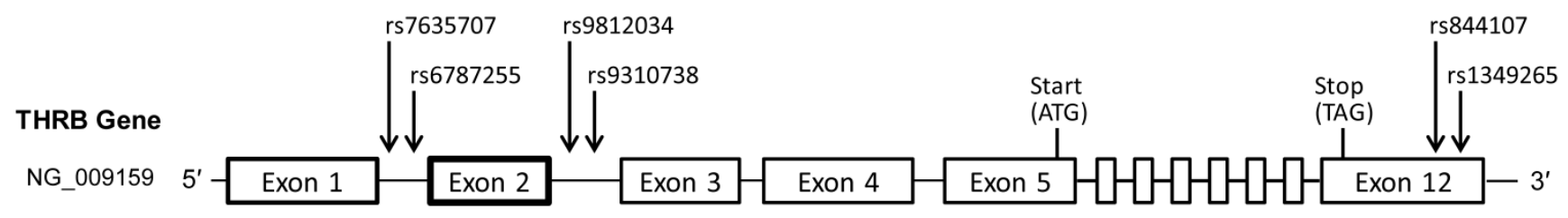

\section{Transcript variant}

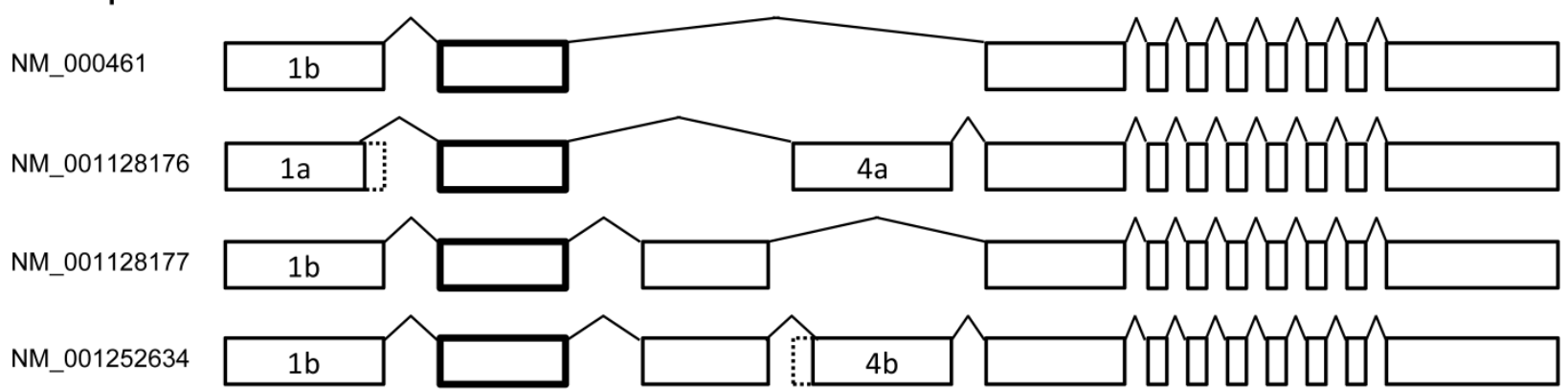

Figure 2. Genomic organization and transcript variants of human THRB genes. The 4 intronic SNPs and 2 SNPs in the 3'-UTR tested are indicated by arrows. 


\section{Results}

\section{Patient characteristics}

Patient characteristics are presented in Table 1. The mean age was $64.5 \pm 7.4$ years (median age, 65 years; range, $48-78$ years). Twenty-four, 20 , and 4 patients showed ECOG PS of 0,1 , and 2, respectively. The PS of 1 patient was not precisely known; however, the PS of this patient was considered to be 0 or 1 , based on the daily activity information from medical records. None of the subjects exhibited clinical manifestations of hypo- or hyperthyroidism that would necessitate medication, although precise laboratory data, including free triiodothyronine (T3), free thyroxine (T4), and $\mathrm{TH}$-stimulating hormone (TSH), were not routinely examined.

Table I. Demographic and clinicopathologic characteristics of 49 patients with esophageal squamous cell carcinoma.

\begin{tabular}{ll}
\hline Age $(\mathrm{y})$ & $64.5 \pm 7.4(48-78)^{\mathrm{a})}$ \\
Height $(\mathrm{cm})$ & $163.5 \pm 6.6(150-180)^{\mathrm{a})}$ \\
Weight $(\mathrm{kg})$ & $56.1 \pm 9.6(33-79)^{\mathrm{a})}$ \\
Sex & Male/Female $=46 / 3$ \\
Performance status & $0 / 1 / 2 /$ unknown $=24 / 20 / 4 / 1$ \\
Tumor differentiation & Well/moderate/poor $/$ unknown $=$ \\
& $7 / 28 / 8 / 6$ \\
TNM $^{\text {b) }}$ & $\mathrm{T} 1 / \mathrm{T} 2 / \mathrm{T} 3 / \mathrm{T} 4=16 / 6 / 15 / 12$ \\
& $\mathrm{~N} 0 / \mathrm{N} 1=22 / 27$ \\
& $\mathrm{M} 0 / \mathrm{M} 1=41 / 8$ \\
Stage & $\mathrm{I} / \mathrm{II} / \mathrm{III} / \mathrm{IV}=12 / 10 / 19 / 8$
\end{tabular}

a) Values represent mean $( \pm S D)$, with the range in parentheses.

b) TNM, tumor node metastasis.

\section{Genotypes, allele frequencies, and haplotypes}

Genotype and allele frequencies are shown in Table 2. The genotype frequencies of wild-type ho- mozygotes, heterozygotes, and homozygote variants of the SNPs rs7635707, rs6787255, rs9812034, and rs9310738 were $29 / 49,16 / 49$, and 4/49, respectively. Frequencies of the CC, CT, and TT genotypes and C allele of the SNP rs844107 were 21/49, 19/49, 9/49, and 61/98, respectively. Frequencies of the GG, GA, and AA genotypes and G allele of the SNP rs1349265 were $27 / 49,18 / 49,4 / 49$, and $72 / 98$, respectively. Given that the genotype frequencies of the 4 intronic SNPs were similar, linkage disequilibrium of these SNPs was additionally examined. Table 3 shows the estimated frequencies of THRB haplotypes; 5 haplotypes were statistically inferred with a frequency $>0.1 \%$ at the population base. With the major 2 haplotypes, the frequency reached approximately $97 \%$ : G-A-G-C (73.4\%) and T-C-T-T (23.5\%). T-A-G-C $(1.0 \%)$, G-C-G-C (1.0\%), and G-A-T-T $(1.0 \%)$ were rare, and other haplotypes were not statistically inferred with a frequency $>0.1 \%$ in the present population.

\section{Severe acute toxicities}

Episodes of severe acute leukopenia, stomatitis, and cheilitis occurred in $42.9 \%$ (21/49), 14.3\% (7/49), and $16.3 \%(8 / 49)$ of cases, respectively. The genotype and allele frequencies of THRB with or without severe acute toxicities are presented in Table 4 . The 4 intronic SNPs, but not the 2 SNPs in the 3'-UTR, exhibited a significant predictive value for severe acute leukopenia. Subjects with the GG genotype of rs7635707 SNP, the AA genotype of rs6787255, the GG genotype of rs9812034, and the CC genotype of rs9310738 were at a lower risk of developing severe acute leukopenia. None of the SNPs analyzed has a relationship with severe acute stomatitis and cheilitis. Analysis of the relationship between leukopenia and haplotypes revealed a lower rate of leukopenia in subjects with the G-A-G-C haplotype.

Table 2. Genotype and allele frequencies of THRB in 49 patients with esophageal squamous cell carcinoma.

\begin{tabular}{|c|c|c|c|c|c|c|c|}
\hline \multicolumn{8}{|c|}{ SNP } \\
\hline \multirow[t]{2}{*}{ dbSNP (NCBI) } & \multirow[t]{2}{*}{ Location } & \multicolumn{3}{|c|}{ allele } & \multicolumn{3}{|c|}{ genotype } \\
\hline & & allele $1 / 2$ & 1 & 2 & $1 / 1$ & $1 / 2$ & $2 / 2$ \\
\hline rs7635707 & intron 1 & $\mathrm{G} / \mathrm{T}$ & 74 & 24 & 29 & 16 & 4 \\
\hline rs6787255 & & $\mathrm{A} / \mathrm{C}$ & 74 & 24 & 29 & 16 & 4 \\
\hline rs9812034 & intron 2 & $\mathrm{G} / \mathrm{T}$ & 74 & 24 & 29 & 16 & 4 \\
\hline rs9310738 & & $\mathrm{C} / \mathrm{T}$ & 74 & 24 & 29 & 16 & 4 \\
\hline rs844107 & 3'-UTR & $\mathrm{C} / \mathrm{T}$ & 61 & 37 & 21 & 19 & 9 \\
\hline rs1349265 & & $\mathrm{G} / \mathrm{A}$ & 72 & 26 & 27 & 18 & 4 \\
\hline
\end{tabular}

THRB, thyroid hormone receptor beta; SNP, single nucleotide polymorphism; dbSNP, the National Center for Biotechnology Information (NCBI) variation database; UTR, untranslated region. 
Table 3. Estimated frequencies of THRB haplotypes in 49 patients with esophageal squamous cell carcinoma.

\begin{tabular}{ccccc}
\hline & \multicolumn{2}{c}{ Intronic SNPs across exon 2 } & \multicolumn{2}{c}{ Estimated Frequency } \\
\hline rs7635707 & rs6787255 & rs9812034 & rs9310738 & N $=49$ \\
\hline G & A & G & C & 0.7343 \\
T & C & T & T & 0.2346 \\
T & A & G & C & 0.0102 \\
G & C & G & C & 0.0102 \\
G & A & T & T & 0.0099 \\
\hline
\end{tabular}

THRB, thyroid hormone receptor beta; SNP, single nucleotide polymorphism.

Table 4. Effects of THRB genetic polymorphisms on severe acute toxicities in 49 patients with esophageal squamous cell carcinoma.

\begin{tabular}{|c|c|c|c|c|c|c|c|c|c|c|c|}
\hline \multirow[t]{3}{*}{ dbSNP (NCBI) } & \multirow[t]{3}{*}{ Location } & & \multicolumn{2}{|c|}{ Leukopenia } & & \multicolumn{2}{|c|}{ Stomatitis } & & \multicolumn{2}{|c|}{ Cheilitis } & \\
\hline & & & yes & no & & yes & no & & yes & no & \\
\hline & & & 21 & 28 & $P$-value & 7 & 42 & $P$-value & 8 & 41 & $P$-value \\
\hline \multirow[t]{5}{*}{ rs7635707 } & \multirow[t]{10}{*}{ intron 1} & GG & 7 & 22 & 0.004 & 5 & 24 & NS & 4 & 25 & NS \\
\hline & & GT & 11 & 5 & & 2 & 14 & & 3 & 13 & \\
\hline & & $\mathrm{TT}$ & 3 & 1 & & 0 & 4 & & 1 & 3 & \\
\hline & & G & 25 & 49 & 0.002 & 12 & 62 & NS & 11 & 63 & NS \\
\hline & & $\mathrm{T}$ & 17 & 7 & & 2 & 22 & & 5 & 19 & \\
\hline \multirow[t]{5}{*}{ rs6787255 } & & AA & 7 & 22 & 0.004 & 5 & 24 & NS & 3 & 26 & NS \\
\hline & & $\mathrm{AC}$ & 11 & 5 & & 2 & 14 & & 4 & 12 & \\
\hline & & $\mathrm{CC}$ & 3 & 1 & & 0 & 4 & & 1 & 3 & \\
\hline & & $\mathrm{A}$ & 25 & 49 & 0.002 & 12 & 62 & NS & 10 & 64 & NS \\
\hline & & $\mathrm{C}$ & 17 & 7 & & 2 & 22 & & 6 & 18 & \\
\hline \multirow[t]{5}{*}{ rs9812034 } & \multirow[t]{5}{*}{ intron 2} & GG & 7 & 22 & 0.004 & 4 & 25 & NS & 3 & 26 & NS \\
\hline & & GT & 11 & 5 & & 3 & 13 & & 4 & 12 & \\
\hline & & $\mathrm{TT}$ & 3 & 1 & & 0 & 4 & & 1 & 3 & \\
\hline & & G & 25 & 49 & 0.002 & 11 & 63 & NS & 10 & 64 & NS \\
\hline & & $\mathrm{T}$ & 17 & 7 & & 3 & 21 & & 6 & 18 & \\
\hline \multirow[t]{5}{*}{ rs9310738 } & & $\mathrm{CC}$ & 7 & 22 & 0.004 & 4 & 25 & NS & 3 & 26 & NS \\
\hline & & CT & 11 & 5 & & 3 & 13 & & 4 & 12 & \\
\hline & & $\mathrm{TT}$ & 3 & 1 & & 0 & 4 & & 1 & 3 & \\
\hline & & $\mathrm{C}$ & 25 & 49 & 0.002 & 11 & 63 & NS & 10 & 64 & NS \\
\hline & & $\mathrm{T}$ & 17 & 7 & & 3 & 21 & & 6 & 18 & \\
\hline \multirow[t]{5}{*}{ rs844107 } & 3'-UTR & $\mathrm{CC}$ & 10 & 11 & NS & 2 & 19 & NS & 3 & 18 & NS \\
\hline & & $\mathrm{CT}$ & 6 & 13 & & 4 & 15 & & 4 & 15 & \\
\hline & & TT & 5 & 4 & & 1 & 8 & & 1 & 8 & \\
\hline & & $\mathrm{C}$ & 26 & 35 & NS & 8 & 53 & NS & 10 & 51 & NS \\
\hline & & $\mathrm{T}$ & 16 & 21 & & 6 & 31 & & 6 & 31 & \\
\hline \multirow[t]{5}{*}{ rs1349265 } & & AA & 1 & 3 & NS & 1 & 3 & NS & 0 & 4 & NS \\
\hline & & AG & 9 & 9 & & 7 & 11 & & 2 & 16 & \\
\hline & & GG & 16 & 11 & & 13 & 14 & & 5 & 22 & \\
\hline & & $\mathrm{A}$ & 11 & 15 & NS & 9 & 17 & NS & 2 & 24 & NS \\
\hline & & G & 41 & 31 & & 33 & 39 & & 12 & 60 & \\
\hline
\end{tabular}

Episodes of leukopenia, stomatitis, and cheilitis during the first 2 courses and the subsequent 2 weeks (until day 70) were recorded as acute toxicities and those of grade 3 or higher, as severe acute toxicities. Statistical significance was assessed with Fisher's exact test. dbSNP, the National Center for Biotechnology Information (NCBI) variation database; NS, not significant. 


\section{Discussion}

Administration of chemotherapy without severe toxicities is of great importance to medical oncologists. In the treatment of ESCC, definitive 5-FU/CDDP-based CRTs are promising modality with organ preservation; however, oncologists have noted that some proportion of patients will be exposed to a high risk of severe toxicities without long-term survival. In this regimen, the most common and alarming one is leukopenia, and 8 to $40 \%$ of patients show grade $3 / 4$ leukopenia [5-7]. In the current study, we demonstrated that ESCC patients with the GG genotype of the THRB rs7635707 SNP, the AA genotype of rs6787255, the GG genotype of rs9812034, and the CC genotype of rs9310738 have a lower probability of severe acute leukopenia than those with the other genotypes.

Germline mutations in THRB have been identified in association with resistance to thyroid hormone, which is quite a rare disease [36]. Such patients harbor missense mutations or defects that correspond to amino acid alterations in the ligand-binding domain [36]. Somatic mutations and aberrant expression have been detected in cancers of different organs, including breast, thyroid, kidney, lung, liver, and colon, suggesting a suppressive role in carcinogenesis and metastasis [37, 38]. However, whether non-coding SNPs in THRB would is still unclear. The present study is the first to demonstrate that the intronic SNPs in THRB could have a clinical impact.

The 4 intronic SNPs analyzed are closely linked together. Given the burden of cost and time, analysis of only one of the 4 intronic SNPs can be a strong predictor of severe acute leukopenia. Analyses of the haplotype frequencies of these SNPs revealed 2 main haplotypes, indicating linkage disequilibrium of the 4 SNPs. However, the analysis did not have enough statistical power to detect the significant association between haplotype or diplotype distribution and severe acute leukopenia. These SNPs were located across exon 2, which is common among the THRB transcript variants. Polymorphism of gene regulatory regions is likely to be one of the major contributors to phenotypic variation between and within human populations [39]. Therefore, this region is considered to be important for the regulation of TR $\beta$ mRNA and protein expression, although the data are limited [12, $40]$. An intron control region regulates TR $\beta 2$ expression in the central nervous system [12], and 3'- and 5'-UTR variants regulate TR $\beta 1$ expression in renal cell cancer [41], but this region is not mentioned in these reports. The precise mechanism should be clarified in future studies.
To date, translational control of TR $\beta$ is believed to regulate cell proliferation, metabolism, and cellular stress responses [14, 42, 43]. These functions may have direct or indirect influence on the severity of chemotherapy-related toxicities. Although the 4 intronic SNPs analyzed showed a strong association with severe acute myelotoxicity, the effects of these SNPs on the expression, regulation, and biological functions of TR $\beta$ are unknown. A large number of THRB SNPs have been identified; however, information on TR $\beta$ mRNA processing, including transcriptional initiation, and expression and function in each tissue and organ according to SNPs is limited [12,44], and the molecular mechanisms underlying the effects of these SNPs remain unclear. Based on the limited available data, the mechanism may be explained as follows. First, the proliferation of hematopoietic stem cells may be directly dependent on these SNPs, given the change in TR $\beta$ expression in these cells. Second, TR $\beta$ may have a variable influence on the phosphatidylinositol 3-kinase (PI3K)-Src homology 2-containing inositol phosphatase-1 (SHIP1) interaction, depending on the SNPs. SHIP1 regulates myeloid cell proliferation, which is controlled by PI3K [45]. PI3K also plays a role in TR $\beta$-regulated cellular survival and proliferation $[46,47]$. Finally, aberrant TR $\beta$ expression in liver and kidney may influence the pharmacokinetics of 5-FU and CDDP [21-24].

Thyroid function should also be considered when analyzing myeloid function [15-18]. In this study, TH-related parameters were not routinely evaluated because no clinical manifestations were found. We believe that subclinical TH disorders will not eradicate the strong impact of THRB SNPs on leukopenia. It is also necessary to consider the influence on thyroid function because irradiation fields often include a part of the thyroid gland. Although it is well known that radiation exposure of the thyroid gland can induce hypothyroidism, its potential influence can be ignored in this study because radiation-induced hypothyroidism would be detected several years after completion of irradiation [48]. Furthermore, possible influence of CDDP-based chemotherapy on thyroid function has been reported in children [49].

Our study has some limitations. First, this is a retrospective study. Second, our pilot study was limited by small sample size and a lack of racial and ethnic diversity. The THRB genotype and haplotype frequencies in healthy subjects also remain to be estimated. Further, detailed studies are needed to identify the mechanisms underlying transcriptional regulation and examine the involvement of other possible factors, including related genotypes and the presence 
of transcription factors, in these mechanisms. Finally, our exploratory reconnaissance selected only 6 candidate SNPs based on the selection criteria described above, and the criteria for selecting useful SNPs from a large number of SNPs remain unclear. Despite these limitations, the present data contribute significant information on the predictive value of SNPs in leukopenia and may prove to be useful tools for individualizing ESCC treatment strategies.

\section{Conclusion}

Our pilot study shows the potential utility of THRB polymorphisms in ESCC patients receiving a definitive 5-FU/CDDP-based CRT, suggesting that specific SNPs in THRB provide pre-treatment warning of severe acute myelotoxicity. The clinical utility of THRB genotyping should be confirmed in prospective studies.

\section{Authors' contributions}

TA, SM, MH, and TS conceived, designed, and coordinated the study. IM, TT, TO, and HO evaluated the clinical outcome. MY and KN determined the THRB SNPs. AK, MY, KK, and KN performed data management and statistical analysis. IM and TM prepared the manuscript. All authors read and approved the final manuscript.

\section{Acknowledgments}

This work was supported in part by a Grant-in-Aid for Young Scientists from the Japan Society for the Promotion of Science.

\section{Competing Interests}

The authors have no competing interests.

\section{References}

1. [Internet] Cancer statistics in Japan. http://ganjoho.ncc.go.jp/public/ statistics/backnumber/2010_en.html.

2. Hongo M, Nagasaki Y, Shoji T. Epidemiology of esophageal cancer: Orient to Occident. Effects of chronology, geography and ethnicity. J Gastroenterol Hepatol. 2009; 24: 729-735.

3. Sakaeda T, Yamamori M, Kuwahara A, et al. Pharmacokinetics and pharmacogenomics in esophageal cancer chemoradiotherapy. Adv. Drug Deliv. Rev. 2009; 61: 388-401.

4. Kranzfelder M, Buchler P, Lange $K$, et al. Treatment options for squamous cell cancer of the esophagus: a systematic review of the literature. J. Am. Coll. Surg. 2010; 210: 351-359.

5. Ohtsu A, Boku N, Muro K, et al. Definitive chemoradiotherapy for T4 and/or M1 lymph node squamous cell carcinoma of the esophagus. J. Clin. Oncol. 1999; 17: 2915-2921.

6. Kaneko K, Ito H, Konishi K, et al. Definitive chemoradiotherapy for patients with malignant stricture due to $\mathrm{T} 3$ or T4 squamous cell carcinoma of the oesophagus. Br. J. Cancer. 2003; 88: 18-24.

7. Kato K, Muro K, Minashi K, et al. Phase II study of chemoradiotherapy with 5-fluorouracil and cisplatin for Stage II-III esophageal squamous cell carcinoma: JCOG trial (JCOG 9906). Int. J. Radiat. Oncol. Biol. Phys. 2011; 81: 684-690.

8. Huang RS, Ratain MJ. Pharmacogenetics and pharmacogenomics of anticancer agents. CA Cancer J. Clin. 2009; 59: 42-55.
9. O'Donnell PH, Ratain MJ. Germline pharmacogenomics in oncology: decoding the patient for targeting therapy. Mol Oncol. 2012; 6: 251-259.

10. Cheng SY, Leonard JL, Davis PJ. Molecular aspects of thyroid hormone actions. Endocr. Rev. 2010; 31: 139-70.

11. Moreno M, de Lange $P$, Lombardi A, et al. Metabolic effects of thyroid hormone derivatives. Thyroid. 2008; 18: 239-253.

12. Jones I, $\mathrm{Ng} \mathrm{L}$, Liu H, Forrest $\mathrm{D}$. An intron control region differentially regulates expression of thyroid hormone receptor beta2 in the cochlea, pituitary, and cone photoreceptors. Mol. Endocrinol. 2007; 21: 1108-1119.

13. Yen PM. Physiological and molecular basis of thyroid hormone action. Physiol. Rev. 2001; 81: 1097-1142.

14. Kress E, Samarut J, Plateroti M. Thyroid hormones and the control of cell proliferation or cell differentiation: paradox or duality? Mol. Cell Endocrinol. 2009; 313: 36-49.

15. Axelrod AR, Berman L. The bone marrow in hyperthyroidism and hypothyroidism. Blood. 1951; 6: 436-453.

16. Foster MP, Montecino-Rodriguez E, Dorshkind K. Proliferation of bone marrow pro-B cells is dependent on stimulation by the pituitary/thyroid axis. J. Immunol. 1999; 163: 5883-5890.

17. Grymula K, Paczkowska E, Dziedziejko V, et al. The influence of 3,3',5-triiodo-L-thyronine on human haematopoiesis. Cell Prolif. 2007; 40: 302-315.

18. Kawa MP, Grymula K, Paczkowska E, et al. Clinical relevance of thyroid dysfunction in human haematopoiesis: biochemical and molecular studies. Eur. J. Endocrinol. 2010; 162: 295-305.

19. Cheng SY. Multiple mechanisms for regulation of the transcriptional activity of thyroid hormone receptors. Rev. Endocr. Metab. Disord. 2000; 1: 9-18.

20. Matsuse M, Saenko V, Sedliarou I, et al. A novel role for thyroid hormone receptor beta in cellular radiosensitivity. J. Radiat. Res (Tokyo). 2008; 49: 17-27.

21. Kowalik MA, Perra A, Pibiri M, et al. TRbeta is the critical thyroid hormone receptor isoform in T3-induced proliferation of hepatocytes and pancreatic acinar cells. J. Hepatol. 2010; 53: 686-692.

22. Lopez-Fontal R, Zeini M, Traves PG, et al. Mice lacking thyroid hormone receptor Beta show enhanced apoptosis and delayed liver commitment for proliferation after partial hepatectomy. PLoS One. 2010; 5: e8710.

23. Flores-Morales A, Gullberg H, Fernandez L, et al. Patterns of liver gene expression governed by TRbeta. Mol. Endocrinol. 2002; 16: 1257-1268.

24. Ornellas Ddos S, Gomes AC, Santiago LA, et al. Thyroid hormone beta receptor mutation causes renal dysfunction and impairment of $\mathrm{ClC}-2$ chloride channel expression in mouse kidney. Cell Physiol. Biochem. 2010; 26: 227-234

25. Plevová P. Prevention and treatment of chemotherapy- and radiotherapy-induced oral mucositis: a review. Oral Oncol. 1999; 35: 453-470.

26. Kosugi S, Sasamoto R, Kanda T, et a. Retrospective review of surgery and definitive chemoradiotherapy in patients with squamous cell carcinoma of the thoracic esophagus aged 75 years or older. Jpn. J. Clin. Oncol. 2009; 39: 360-366.

27. Miki I, Tamura T, Nakamura T, et al. Circadian variability of pharmacokinetics of 5-fluorouracil and CLOCK T3111C genetic polymorphism in patients with esophageal carcinoma. Ther. Drug Monit. 2005; 27: 369-374.

28. Okuno T, Tamura T, Yamamori $\mathrm{M}$, et al. Favorable genetic polymorphisms predictive of clinical outcome of chemoradiotherapy for stage II/III esophageal squamous cell carcinoma in Japanese. Am. J. Clin. Oncol. 2007; 30: 252-257.

29. Sakaeda T, Yamamori M, Kuwahara A, et al. VEGF G-1154A is predictive of severe acute toxicities during chemoradiotherapy for esophageal squamous cell carcinoma in Japanese patients. Ther. Drug Monit. 2008; 30: 497-503.

30. Kuwahara A, Yamamori M, Fujita M, et al. TNFRSF1B A1466G genotype is predictive of clinical efficacy after treatment with a definitive 5 -fluorouracil/cisplatin-based chemoradiotherapy in Japanese patients with esophageal squamous cell carcinoma. J. Exp. Clin. Cancer Res. 2010; 29: 100 .

31. [Internet] dbSNP. http://www.ncbi.nlm.nih.gov/projects/SNP/.

32. Stephens M, Smith NJ, Donnelly P. A new statistical method for haplotype reconstruction from population data. Am. J. Hum. Genet. 2001; 68: 978-89.

33. Stephens M, Donnelly P. A comparison of bayesian methods for haplotype reconstruction from population genotype data. Am. J. Hum. Genet. 2003; 73: 1162-1169.

34. Komoto C, Nakamura T, Sakaeda T, et al. MDR1 haplotype frequencies in Japanese and Caucasian, and in Japanese patients with colorectal cancer and esophageal cancer. Drug Metab. Pharmacokinet. 2006; 21 : 126-132. 
35. Markova S, Nakamura T, Makimoto H, et al. IL-1beta genotype-related effect of prednisolone on IL-1beta production in human peripheral blood mononuclear cells under acute inflammation. Biol. Pharm. Bull. 2007; 30: 1481-1487.

36. Refetoff S, Dumitrescu AM. Syndromes of reduced sensitivity to thyroid hormone: genetic defects in hormone receptors, cell transporters and deiodination. Best Pract. Res. Clin. Endocrinol. Metab. 2007; 21: 277-305.

37. Gonzalez-Sancho JM, Garcia V, Bonilla F, et al. Thyroid hormone receptors/THR genes in human cancer. Cancer Lett. 2003; 192: 121-132.

38. Aranda A, Martinez-Iglesias O, Ruiz-Llorente L, et al. Thyroid receptor: roles in cancer. Trends Endocrinol. Metab. 2009; 20: 318-324.

39. Pastinen T, Hudson TJ. Cis-acting regulatory variation in the human genome. Science. 2004; 306: 647-650

40. Frankton S, Harvey CB, Gleason LM, et al. Multiple messenger ribonucleic acid variants regulate cell-specific expression of human thyroid hormone receptor beta1. Mol. Endocrinol. 2004; 18: 1631-1642.

41. Master A, Wojcicka A, Piekielko-Witkowska A, et al. Untranslated regions of thyroid hormone receptor beta 1 mRNA are impaired in human clear cell renal cell carcinoma. Biochim. Biophys. Acta. 2010; 1802: 995-1005.

42. Puzianowska-Kuznicka M, Pietrzak M, Turowska O, et al. Thyroid hormones and their receptors in the regulation of cell proliferation. Acta. Biochim. Pol. 2006; 53: 641-650.

43. Amorim BS, Ueta CB, Freitas BC, et al. A TRbeta-selective agonist confers resistance to diet-induced obesity. J. Endocrinol. 2009; 203: 291-299.

44. Alberobello AT, Congedo V, Liu H, et al. An intronic SNP in the thyroid hormone receptor beta gene is associated with pituitary cell-specific over-expression of a mutant thyroid hormone receptor beta2 (R338W) in the index case of pituitary-selective resistance to thyroid hormone. J. Transl. Med. 2011; 9: 144

45. Gloire G, Erneux C, Piette J. The role of SHIP1 in T-lymphocyte life and death. Biochem. Soc. Trans. 2007; 35: 277-280.

46. Moeller LC, Haselhorst NE, Dumitrescu AM, et al. Stanniocalcin 1 induction by thyroid hormone depends on thyroid hormone receptor beta and phosphatidylinositol 3-kinase activation. Exp. Clin. Endocrinol. Diabetes. 2011; 119: 81-85.

47. Furuya F, Lu C, Guigon CJ, et al. Nongenomic activation of phosphatidylinositol 3-kinase signaling by thyroid hormone receptors. Steroids. 2009; 74: 628-634.

48. Boomsma MJ, Bijl HP, Langendijk JA. Radiation-induced hypothyroidism in head and neck cancer patients: a systematic review. Radiother. Oncol. 2011; 99: 1-5.

49. van Santen HM, Thonissen NM, de Kraker J, et al. Changes in thyroid hormone state in children receiving chemotherapy. Clin. Endocrinol (Oxf). 2005; 62: 250-257 